Scientia Marina 74(2)

June 2010, 371-384, Barcelona (Spain)

ISSN: 0214-8358

doi: 10.3989/scimar.2010.74n2371

\title{
Consideration of uncertainty in the design and use of harvest control rules
}

\author{
YAN JIAO ${ }^{1}$, KEVIN REID $^{2}$ and TOM NUDDS ${ }^{3}$ \\ ${ }^{1}$ Department of Fisheries and Wildlife Sciences, Virginia Polytechnic Institute and State University, Blacksburg, \\ VA, 24061-0321, U.S.A. E- mail: yjiao@vt.edu \\ ${ }^{2}$ Ontario Commercial Fisheries Association, Box 2129, 45 James Street, Blenheim, ON, NOP 1A0, Canada. \\ ${ }^{3}$ Department of Integrative Biology, University of Guelph, Guelph, ON, N1G 2W1, Canada.
}

SUMMARY: Harvest control rules are widely used by management agencies for decision-making and for promoting public awareness of the status of marine and freshwater fisheries. Many current control rules combine fishing mortality and biomass-based biological reference points. Control rules were introduced as a precaution against the influence of uncertainty and to decrease the risk of overfishing, but are compromised if the uncertainties of the biological reference points are not explicitly considered. Uncertainty has been widely acknowledged but has not been incorporated into control rule design and application. In this paper, we used a Bayesian statistical catch-at-age model to estimate uncertainties in the indicators of fishing mortality, population size, and biological reference points. We apply this model to the Lake Erie walleye (Sander vitreus) fishery, and by fully considering the uncertainty of the indicators, the risk of overfishing and the risk of the population being overfished can be explicitly estimated in the control rules. We suggest short and long-term approaches to incorporate uncertainty in the design of control rules. We also suggest that control rules for specific fisheries should be designed with explicit consideration of the uncertainty of the biological reference points, based on a risk level that the management agency and stakeholders agree upon.

Key words: harvest control rule, fishery status evaluation, uncertainty, decision-making, Bayesian analysis.

RESUMEN: INCORPORACIÓN DE LA INCERTIDUMBRE EN EL DISEÑO Y APLICACIÓN DE REGLAS DE CONTROL DE PRODUCCIÓN. - Las reglas de control de producción se usan generalmente por los organismos de gestión de recursos pesqueros para tomar decisiones y para promover la concienciación pública sobre el estado de conservación de pesquerías marinas y de aguas dulces. Muchas de las reglas de control actuales combinan puntos de referencia biológicos basados en los indicadores mortalidad por pesca y biomasa. Las reglas de control fueron introducidas como precaución contra la influencia de la incertidumbre en las evaluaciones y para reducir el riesgo de sobrepesca, pero se ven comprometidas si la incertidumbre en los puntos de referencia biológicos no se consideran explícitamente. El papel de la incertidumbre se reconoce ampliamente, pero no ha sido incorporado hasta ahora en el diseño y aplicación de reglas de control. En la presente contribución se aplica un modelo bayesiano de capturas por edad para estimar la incertidumbre en los indicadores de mortalidad por pesca, tamaño de la población y puntos de referencia biológicos. Se aplica este modelo a la pesquería de Sander vitreus del Lago Erie, y, mediante la completa incorporación de la incertidumbre de los indicadores, el riesgo de sobrepesca puede ser estimado explícitamente en las reglas de control. Se sugieren aproximaciones a corto y largo plazo para la incorporación de la incertidumbre en el diseño de reglas de control. Se sugiere también que las reglas para pesquerías particulares deben diseñarse incorporando explícitamente la incertidumbre en los puntos de referencia biológicos, en base al nivel de riesgo que se acuerde entre el organismo encargado de la gestión del recurso y sus usuarios.

Palabras clave: regla de control de producción, evaluación del estado de las pesquerías, incertidumbre, toma de decisiones, análisis bayesiano. 


\section{INTRODUCTION}

Harvest control rules are widely used by fisheries management agencies of the United States and other countries for decision-making, and for promoting public awareness of the status of marine and freshwater fisheries (Restrepo et al., 1998; Restrepo and Powers, 1999). A harvest control rule describes how the harvest is intended to be controlled in relation to the state of an indicator of stock status. Constant catch (such as maximum sustainable yield [MSY]) and constant fishing mortality (such as $F_{m s y}$ and $F_{0.1}$ ) are two approaches of simple harvest control rules (FAO, 1995; Garcia, 1996; Restrepo et al., 1998).

Harvest control rules evolved as fisheries science and management embraced concepts of MSY, precautionary management, and risk assessment. Many current control rules consider multiple biological reference points, such as fishing mortality (F-based biological reference points) and biomass (B-based biological reference points), simultaneously in a single control rule, and implement such biological reference points as target reference points and/or limit reference points (Caddy and Mahon, 1995; Restrepo and Powers, 1999). A target reference point expresses the desired state of a fishery in terms of parameters such as output (catch), fishing effort, or capacity, stated as an explicit management objective for the fishery. A limit reference point (or threshold) is the limit beyond which the state of a fishery is undesirable (Garcia, 1996).

Examples of control rules used in fisheries management are shown in Figure 1 (Butterworth and Best, 1994; SAFMC, 2005; ASMFC, 2002). Control rules (a) and (c) refer only to target reference points, whereas (b) and (d) refer to both target reference points and limit reference points (or thresholds). Control rules (a) and (b) are less conservative than (c) and (d), which have thresholds for low stock biomass, below which the fishery is closed. The state of a particular fishery, with respect to whether it is overfished and whether overfishing is happening, can be thought of graphically as the fishery's location in the region defined by the biological reference points, relative to the target reference point and/or limit reference point expressed in a control rule.

Uncertainty, however, is not incorporated into any of the examples given in Figure 1. Typically, estimates of fishing mortality $\left(F_{m s y}\right)$, biomass, and biomass at maximum sustainable yield $\left(B_{m s v}\right)$ are highly uncertain (Thompson, 1993; Hilborn, 2002;
Katsukawa, 2004). Implementation uncertainty (also known as control uncertainty) is the uncertainty that arises during the process of implementing a fishery management strategy or policy. This uncertainty exists in every fishery with management based on the fishing mortality or quota (FAO, 1995; Francis and Shotton, 1997). It may be caused by fishermen not keeping their catches to the quota limits (fishing mortality inadequately controlled). Uncertainty in the fishing mortality or quota can arise when the quota has been set inaccurately (i.e. fishing mortality inadequately assessed because of deficiencies in model selection, data quality, etc.). We can find many examples in which Total Allowable Catches (TACs) were exceeded; we can also find many examples in which decisions on fishing mortalities and TACs turned out to be high or low when more new data were included in the model. Fishing mortality is therefore an appropriate basis for determining the above uncertainties if an F-based biological reference point is used in the control rule. If allowable catch or quota is used as a biological reference point in the control rule, uncertainty of the catch or uncertainty of a wrong quota is used in the control rule. However, this has not been explicitly considered in the design and application of control rules. Control rules have evolved in such a way that fisheries managers are increasingly risk averse as a hedge against uncertainty that might lead to overfishing and fishery collapse. But avoidance of one kind of risk entails increasing others (Sunstein, 2005), such as a foregone harvest opportunity, and this can have important economic and social consequences (Hilborn, 2006). Fisheries management might therefore be well served by further development of control rules that formally and explicitly incorporate uncertainty to better assess the risk of overfishing. In turn, this would allow better assessment of a fishery's performance efficiency by evaluating tradeoffs among different types of risk (May et al., 1979; Roughgarden and Smith, 1996).

Recent studies suggested that a general risk assessment approach in fisheries stock assessment should consider uncertainty in both indicators (e.g. fishing mortality $F$ ) and biological reference points (e.g. $F_{m s y}$ ) (Helser et al., 2001; Chen and Wilson 2002; Jiao et al., 2005). However, uncertainty has previously been ignored or not explicitly assessed in fisheries when designing control rules. In this paper, we used data from the Lake Erie walleye (Sander vitreus) fishery and a Bayesian statistical catch-atage model to demonstrate the design and use of con- 
(a)

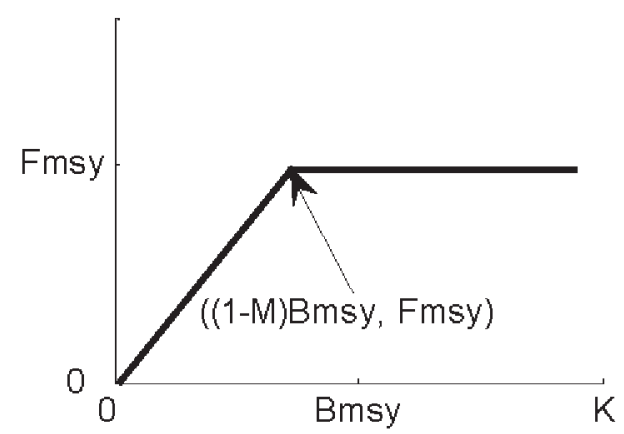

(c)

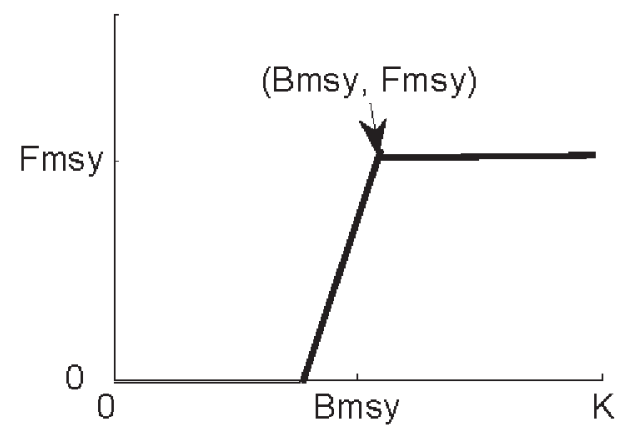

(b)

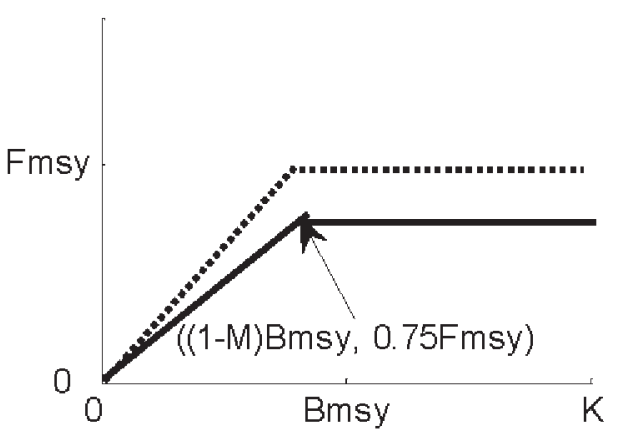

(d)

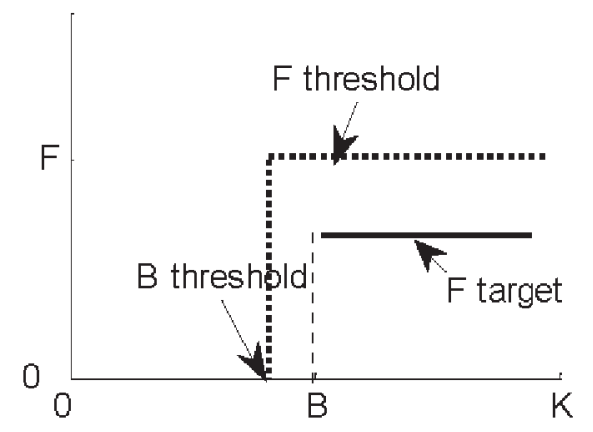

FIG. 1. - Examples of control rules used in fisheries management around the world: (a) south Atlantic black sea bass (Centropristis striata), (b) Atlantic shortfin squid (Illex illecebrosus), (c) Antarctic whales, and (d) Atlantic weakfish (Cynoscion regalis).

trol rules that consider the uncertainty of biological reference points and incorporate the uncertainty of fishing mortality and population size. Specifically, we estimated fishery status as the probability distribution of the fishery existing in different regions of the bivariate region defined by F- and B-based biological reference points (as shown graphically in Fig. 1) (Punt and Hilborn, 1997; Patterson et al., 2001; Chen et al., 2003). We show that control rules can be designed on the basis of specific biological reference points of a fishery, as well as $F$ and $B$ estimation, and that fishery status uncertainty can be built into the control rules in a probabilistic way. The design of a control rule for a specific fishery is based on the risk level that is acceptable to the fishery management agency and stakeholders. Fishing mortality and population size change over time, and this may cause difficulties in incorporating uncertainty when the control rule is designed and explained, since the control rule functions for future management. This study provides a method for building uncertainty related to fishing mortality, into a control rule design based on the previous year's estimate of its uncertainty. By doing this, uncertainty in both indicators and biological reference points are considered in the design of a control rule.

\section{MATERIALS AND METHODS}

\section{Data sources of the example fishery}

Walleye fishery data were obtained from the Ontario Ministry of Natural Resources, Great Lakes Fisheries Commission and the Lake Erie Walleye Task Group (2008). Available data included a time series from 1978 to 2007 of (1) the commercial (gillnet) fishery catch; (2) the recreational (angling) fishery catch; (3) the recreational fishery Catch Per Unit Effort (CPUE), in numbers of fish age 2+ per angler-hour; (4) the commercial fishery CPUE in numbers of fish age $2+$ per km net; and (5) an index of abundance of age 2+ fish from fishery-independent gillnet surveys between 1990 and 2007.

\section{Control rules used as examples in this study}

The Target Limit Control Rule (TLCR, Fig. 1b) was used as an example of how to explicitly consider uncertainty in the control rule, which includes both target and limit reference points (Restrepo et al., 1998). This control rule was proposed by Restrepo et al. (1998, see their Fig. 9) and is widely used in many fisheries. Minimum stock size threshold ( $B_{\text {threshold }}$, 
measured in terms of biomass or abundance) is the minimum safe stock size necessary to maintain or rebuild a fishery, i.e. a biomass limit reference. Maximum fishing mortality threshold ( $F_{\text {threshold }}$ ) is the measurement of fishing mortality that determines whether overfishing is occurring; usually equivalent to $F$ corresponding to the MSY control rule, i.e. a fishing mortality limit reference. Because walleye are managed by numbers (abundance) and not yield (biomass), we modified the control rule of Restrepo et al. (1998) from $S S B_{\text {threshold }}=(1-M)$ $S S N_{m s y}$ to $S S N_{\text {threshold }}=(1-M) S S N_{m s y}$. Here, $M$ is the natural mortality; $S S B_{m s y}$ is the Spawning Stock Biomass at MSY (maximum sustainable yield); and $S S N_{m s y}$ is the spawning stock population abundance of age 2+ fish at MSY. Therefore, $F_{\text {threshold }}=F_{m s y}$ if $S S N>S S N_{\text {threshold }}$; and

$$
F_{\text {threshold }}=F_{\text {msy }} \frac{S S N}{S S N_{\text {threshold }}} \text { if } S S N<S S N_{\text {threshold }} \text {. }
$$

The target fishing mortality rate $\left(F_{\text {target }}\right)$ is set $25 \%$ below the limit fishing mortality (a default TLCR in many fisheries) as shown in Figure 2. The target fishing mortality is $F_{\text {target }}=0.75 F_{m s y}$ if $S S N>$ $S S N_{\text {threshold }}$, and

$$
F_{\text {target }}=0.75 F_{\text {msy }} \frac{S S N}{S S N_{\text {threshold }}} \text { if } S S N<S S N_{\text {threshold }} .
$$

This control rule provides a buffer against fishing mortality exceeding $F_{\text {threshold }}$.

The various regions in Figure 2 can be regarded as hypothesized states of the fishery at any time $t$. For example, for the region labeled H5 the status of the fishery is such that spawning stock abundance is smaller than the $S S N_{\text {threshold }}$ and $F$ is larger than $F_{\text {target }}$ but smaller than $F_{\text {threshold }}\left(F_{m s y}\right)$. The region where a fishery is located can be determined by comparing its $F$ with the $F_{\text {target }}$ and $F_{\text {threshold }}$, and by comparing its estimated spawning stock abundance with the $S S N_{\text {threshold }}$. Stock size is expected to decrease if the fishery lies in any region above the $F / F_{m s y}$ limit line, and to increase if the fishery lies below the target line $\left(F_{\text {target }}\right)$. However, these decreases or increases may not happen as expected because of uncertainty in the dynamics of recruitment and the possibly high population growth rate when the population is small. The limit and target lines are designed to protect against such uncertainty in the biological reference points.

\section{The statistical catch-at-age model}

A statistical catch-at-age model (Hilborn and Walters, 1992; Quinn and Deriso, 1999) based on the available Lake Erie walleye fishery data was written as

$$
\begin{aligned}
& E\left(N_{a+1, y+1}\right)=N_{a, y} e^{-F_{a, y}-M} \\
& E\left(C_{i, a, y}\right)=\frac{F_{i, a, y}}{F_{i, a, y}+M} N_{a, y}\left(1-e^{-F_{i, a, y}-M}\right) \\
& F_{i, a, y}=F_{i, y} S_{i, a} \\
& E\left(I_{j, a, y}\right)=q_{j, a} N_{a, y}
\end{aligned}
$$

where $a$ is age, $y$ is year, $i$ is the $\mathrm{i}^{\text {th }}$ fishery (commercial or recreational; $i=1$ or 2 ), $j$ is the $\mathrm{j}^{\text {th }}$ abundance index (commercial fishery, recreational fishery, or fishery-independent gillnet survey; $j=1,2$, or 3 ), $N_{a, y}$ is the population size of age $a$ fish in year $y, C_{i, a, y}$ is the catch of age $a$ fish in year $y$ by the $\mathrm{i}^{\text {th }}$ fishery, $F_{i, a, y}$ is the fishing mortality rate of age $a$ fish in year $y$ by the $\mathrm{i}^{\text {th }}$ fishery, $S_{i, a}$ is the fishing selectivity of age $a$ fish by the $\mathrm{i}^{\text {th }}$ fishery, and $M=0.32$ is the natural mortality rate (Lake Erie Walleye Task Group, 2004). $I_{j, a, y}$ is the $\mathrm{j}^{\text {th }}$ abundance index observed for age $a$ in year $y$, and $q_{j, a}$ is the catchability coefficient that calibrates the relationship between the $j^{\text {th }}$ abundance index and population abundance

To quantify the uncertainty in this model, we used an observation-process-error estimator, which considers both the process error in the equation of population size $\left(E\left(N_{a+1, y+1}\right)\right)$ and the observation error in the equations of abundance indices $\left(E\left(I_{j, a, y}\right)\right)$ and catch $\left(E\left(C_{i, a, y}\right)\right)$ (Eq. 1). We assumed lognormal error structures for both process error and observation errors, which gave the corresponding objective functions for the estimator as follows. For process error:

$$
\begin{gathered}
\varepsilon_{N} \sim N\left(0, \sigma_{N}^{2}\right) ; l_{N}= \\
=\prod_{y} \prod_{a} \frac{1}{N_{a, y} \sqrt{2 \pi} \sigma_{N}} \exp \left(\frac{-\left[\operatorname{Ln}\left(N_{a, y}\right)-\operatorname{Ln}\left(\hat{N}_{a, y}\right)\right]^{2}}{2 \sigma_{N}^{2}}\right)
\end{gathered}
$$

for measurement error in fishery dependent catch:

$$
\begin{gathered}
l_{i, C}=\prod_{y} \prod_{a} \frac{1}{C_{i, a, y} \sqrt{2 \pi} \sigma_{i, C}} \\
\exp \left(\frac{-\left[\operatorname{Ln}\left(C_{i, a, y}\right)-\operatorname{Ln}\left(\hat{C}_{i, a, y}\right)\right]^{2}}{2 \sigma_{i, C}^{2}}\right)
\end{gathered}
$$


for measurement error in the abundance indices:

$$
\begin{gathered}
l_{j, I}=\prod_{y} \prod_{a} \frac{1}{I_{j, a, y} \sqrt{2 \pi} \sigma_{j, I}} \\
\exp \left(\frac{-\left[\operatorname{Ln}\left(I_{j, a, y}\right)-\operatorname{Ln}\left(\hat{I}_{j, a, y}\right)\right]^{2}}{2 \sigma_{j, I}^{2}}\right) .
\end{gathered}
$$

The total objective function is the sum of (2), (3), and (4) after they are log transformed:

$$
L L=\sum_{i} \operatorname{Ln}\left(l_{i, C}\right)+\sum_{j} \operatorname{Ln}\left(l_{j, I}\right)+\operatorname{Ln}\left(l_{N}\right) .
$$

Lake Erie walleye abundance indices have fluctuated greatly because of the occurrence of a few very strong year classes. Therefore, it is critically important to consider process errors in the model (Eq. 2). Recent research (de Valpine and Hasting, 2002) on the comparison of observation-error and observation-process-error estimators also suggests the importance of using observation-process-error estimators (Eq. 5).

The time series of population size is estimated by projecting the abundance forward from the start of the annual catch series, with the initial abundance $\left(N_{a, 1978}\right)$, recruitment and $F_{i, y}$ and $S_{i, a}$ as parameters (Quinn and Deriso, 1999). Because annual recruitment of walleye was observed to fluctuate dramatically over time, recruitment each year was estimated directly from the statistical catch-at-age model instead of using a built-in stock recruitment relationship. Age-specific selectivity was not modeled using a selectivity curve, but treated as an unknown parameter because it is primarily determined by size preferences of the fishers and anglers (K. Reid, personal observation). The method of Shepherd (1982) was used to estimate $F_{m s y}$ and $S S N_{m s y}$, which combines a yield-per-recruit model with a stock recruitment model. In this study, the abundance of age 3 and older fish is treated as spawning stock abundance, and the abundance of age 2 fish is treated as recruitment. Here we used model-averaged results from Ricker and Beverton-Holt stock recruitment models (Hoeting et al., 1999; Brodziak and Legault, 2005).

\section{Bayesian approach to incorporating uncertainty in the control rules}

Bayesian methods use the Bayes' rule to calculate a "posterior" distribution from the observed data and a "prior" distribution, which is a summary of prior knowledge of the parameters (Rubin, 1988; Smith and Gelfand, 1992; McAllister and Ianelli, 1997). We used numerically intensive WinBUGS software to implement general Bayesian models using "Metropolis-Hasting within Gibbs sampling" (Gilks, 1996; Spiegelhalter et al., 2004), which is particularly useful when dealing with highly dimensional, nonlinear models for Bayesian statistics. A detailed description of the "Metropolis-Hasting within Gibbs sampling" algorithm for process-observation error models can be found in Millar and Meyer (2000).

Bayesian implementation requires specification of prior distributions on all unobserved quantities. Non-informative priors (here, wide uniform distribution) were used for precision parameters, here defined as the reciprocal of the variance of the error terms in the process and observation equations. For the statistical catch-at-age model, selectivity was assumed to be $U(0.3,1)$ (uniform distribution between 0.3 and 1) for both commercial and recreational fisheries. Wide non-informative uniform distributions were used for recruitment, age-specific abundance, and fishing mortality.

A critical issue in using Markov Chain Monte Carlo (MCMC) methods (including MetropolisHasting within Gibbs sampling) is how to determine when random draws have converged to the posterior distribution. We used three methods: monitoring the trace for key parameters; diagnosing the autocorrelation plot for key parameters, and Gelman and Rubin statistics (Spiegelhalter et al., 2004). Three chains were used. After several sets of analyses, for each chain, the first 300000 iterations with a thinning interval of 5 were discarded. Another 100000 iterations for each chain with a thinning interval of 5 were saved and used in the Bayesian analysis, i.e. a total of 300000 iterations were saved. Convergence diagnostics suggest that convergence was achieved at a level that does not compromise our conclusions.

Estimated population abundance and fishing mortality arising from each hypothetical set of parameter values were then compared to evaluate the probability of the fishery being located in each region of Figure 2 (see the schematic of the algorithm in Fig. 3 ). In this way, individual uncertainties of indicators and biological reference points were aggregated to give a clearer understanding of their combined influence on overfishing risk. Uncertainty in fishery status was defined as the probability that the fishery actually lay in one of the areas in the bivariate region describing the control rule (Fig. 2). The uncertainties from $F, F_{m s y}, S S N$, and $S S N_{m s y}$ were all included 

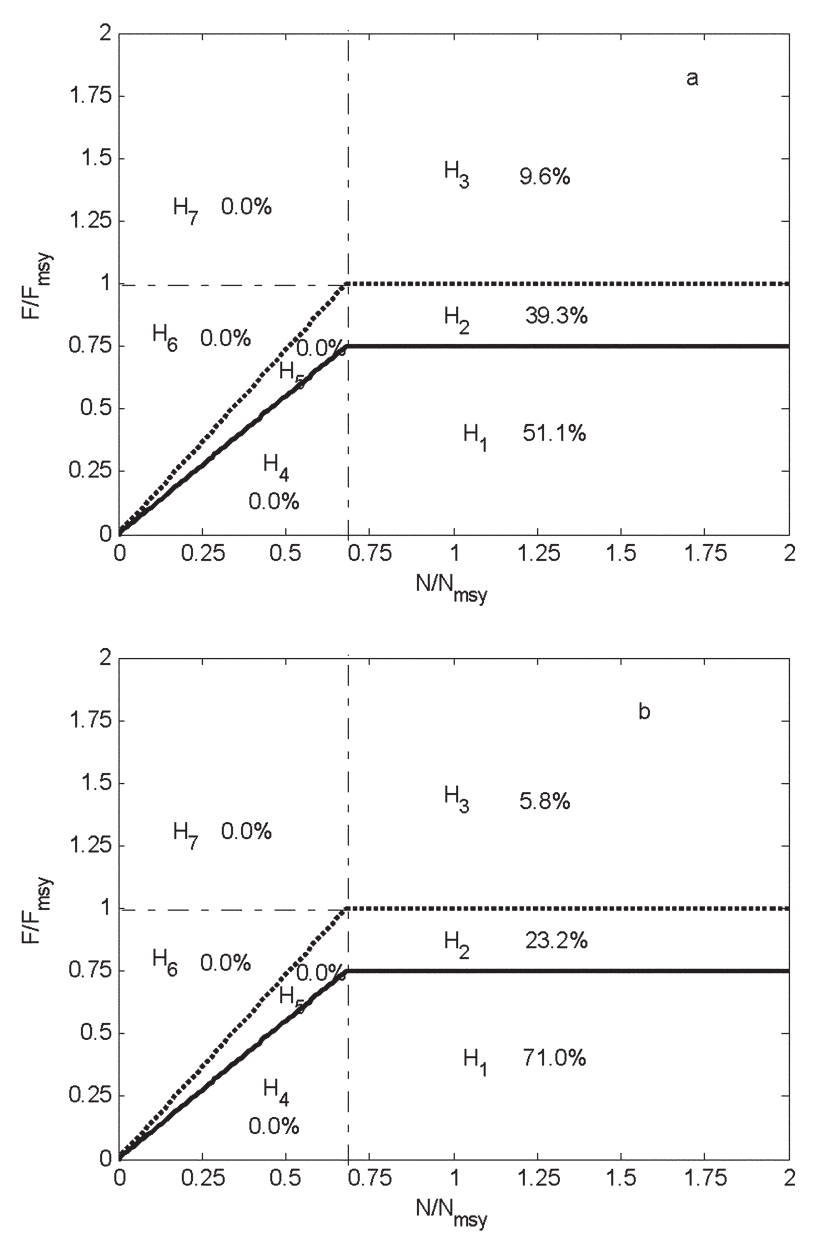

FIG. 2. - Fishery status and its uncertainty shown as the Target Limit Control Rule. Percentages in each region indicate the probability that the fishery was in that state in 2003 (a) and 2007(b). Solid thick line: target reference line; dotted thick line: limit reference line.

when the probabilities of the fishery being located in each of the graphical regions were estimated.

\section{Designing and presenting a control rule with uncertainty considered}

Most control rules are based on experience with specific fisheries to estimate targets for biological reference points (such as fishing mortality and $S S N_{\text {threshold }}$ ) to satisfy the requirement for stock sustainability (e.g. $S S N_{\text {threshold }}=(1-M) S S N_{m s y}$ and $F_{\text {target }}$ $=0.75 F_{m s y}$ ). In this paper, we designed control rules based on the probability distributions of these parameters with a Bayesian approach, i.e. the posterior distributions of fishing mortality, population size, $F_{m s y}$ and $S S N_{m s y}$. Here, we present a risk of $30 \%$ for $F$ larger than $F_{m s y}$ and a risk of $80 \%$ for $S S N$ smaller than $S S N_{m s y}$ as an example of the level of risk accepted by stakeholders. In this example fishery, we incorporated the uncertainty of $\mathrm{F}$ in the projection and the control rule design, so that uncertainties from $F, F_{m s y}, S S N$, and $S S N_{m s y}$ were all considered. We used the uncertainties ( $\mathrm{CV}$ of $F$ ) from the current year as the uncertainties for the following years when we designed the control rule for short-term (1 year to a half generation time or 1-5 years) fishery management. For long-term ( $1 / 2$ to 1 generation time) fishery management, the average of the uncertainties of $F$ in the previous years is used.

In this example fishery, $F, F_{m s y}, S S N$, and $S S N_{m s y}$ were estimated from the same model, and a joint posterior distribution of the population size and all the parameters was distilled in each of their posterior trajectories. The projection procedure can be written as: 1) call the saved Bayesian posterior runs of population size in 2007, the last year that data were available in this example; 2) generate an $F$ probability distribution according to a $\%$ of $F_{m s y}$ with an initial guess of the fraction value from $P\left(F>F_{m s y}\right)$ $=30 \% ; 3$ ) project the population forward to the next year; 4) estimate $P\left(F>F_{m s y}\right)$ and $P\left(S S N<S S N_{m s y}\right)$; 5) repeat steps 1 to 4 by projecting to another year till the target simulation year is reached. During the simulations, $F$ as a fraction of $F_{m s y}$ was adjusted to match the criterion of both $P\left(F>F_{m s y}\right)=30 \%$ and $P\left(S S N<S S N_{m s y}\right)=80 \%$ depending on the management needs. For example, the management objective can be $F \mid$ both $P\left(F>F_{m s y}\right)=30 \%$ and $P(S S N<$ $\left.S S N_{m s y}\right)=80 \%$; or it can be $F \mid P\left(F>F_{m s y}\right)=30 \%$ and $P\left(S S N_{\text {target year }}<S S N_{\text {msy }}\right)=80 \%$. This requires that adjustment of the probability distribution of $F$ is needed, so that it satisfies both the risk of overfishing the fishery and the risk of the population being overfished (see flowchart in Fig. 3).

In many fisheries stock assessments, SSN and $F$ may be estimated from one model, while biomass and $F$-based biological reference points may be estimated from another model; the correlation between $F$ and an $F$-based biological reference point, and the correlation between SSN and a biomass-based biological reference point, are not estimable (Jiao et al., 2005). This implies that a joint distribution of $S S N$, $S S N_{m s y}$ and $F, F_{m s y}$ is not available in both the parameter estimates from the model, and the population projection. We can still use the current and historical years' uncertainties of $F$ (CV here) as the uncertainty for the future population short-term projection. $\mathrm{CV}$ can be used when the future years' control rule is designed for management because it is relatively more stable in magnitude than variances. The distri- 
Do Bayesian analysis based on the MCMC algorithm

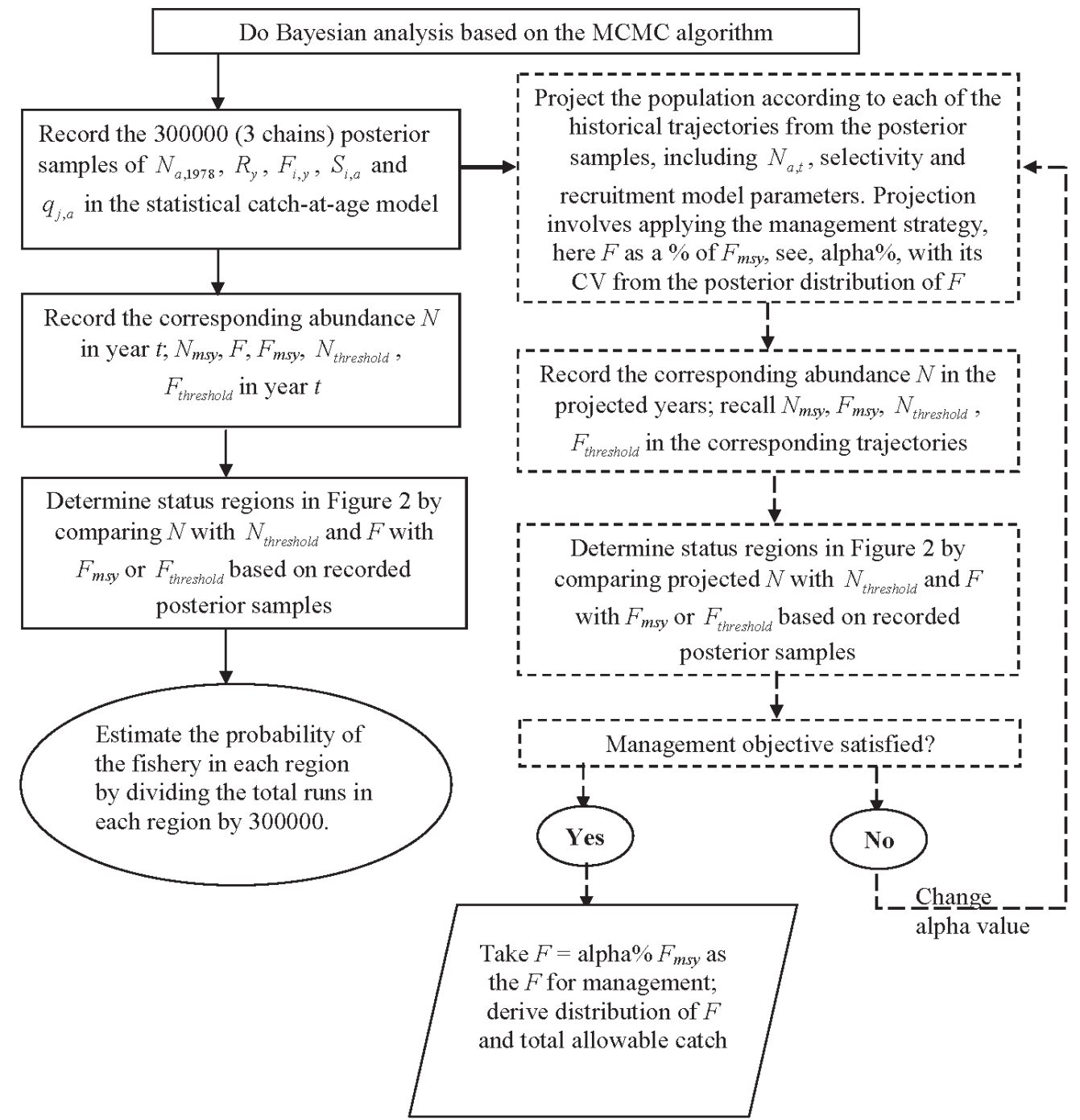

FIG. 3. - Uncertainty estimation algorithm for control rules using a Bayesian method, with 300000 posterior samples to estimate the fishery status uncertainty.

butions of $F_{m s v}$ and $S S N_{m s v}$ are relatively stable and are not sensitive to a couple of new years' data. We showed how to design the control rule in this situation by assuming that $S S N$ and $F$, and $S S N_{m s y}$ and $F_{m s y}$ were from different models for demonstration. A new control rule can be designed by taking into account the uncertainty of $S S N, S S N_{m s y}$ and $F, F_{m s y}$. The projection procedure can be written as: 1) call the saved Bayesian posterior runs of the population size in 2007, the last year that data were available in this example; 2) generate an $F$ probability distribution according to a $\%$ of $F_{m s y}$ with an initial estimate of the fraction value from $\left.P\left(F>F_{m s y}\right)=30 \% ; 3\right)$ project the population forward to the next year; 4) estimate $P\left(F>F_{m s y}\right)$ and $P\left(S S N<S S N_{m s y}\right)$ with appropriate assumptions on all the distributions of $F$,
$F_{m s y}$ and $S S N, S S N_{m s y}$ based on their empirical probability distributions, the correlation between $F$ and $F_{m s y}$, and the correlation between $S S N$ and $S S N_{m s y}$; 5) repeat steps 1 to 4 by projecting to another year till the target simulation year is reached. During the simulations, $F$ as a fraction of $F_{m s y}$ was adjusted to match the criterion of both $P\left(F>F_{m s y}\right)=30 \%$ and $P\left(S S N<S S N_{m s y}\right)=80 \%$ depending on the management needs.

A 5 year projection was carried out based on the example fishery and a $30 \%$ acceptable risk of overfishing. The corresponding catches with uncertainty over time were reported as an example to allow uncertainty including implementation and estimation uncertainty of fishing mortality to be considered. 

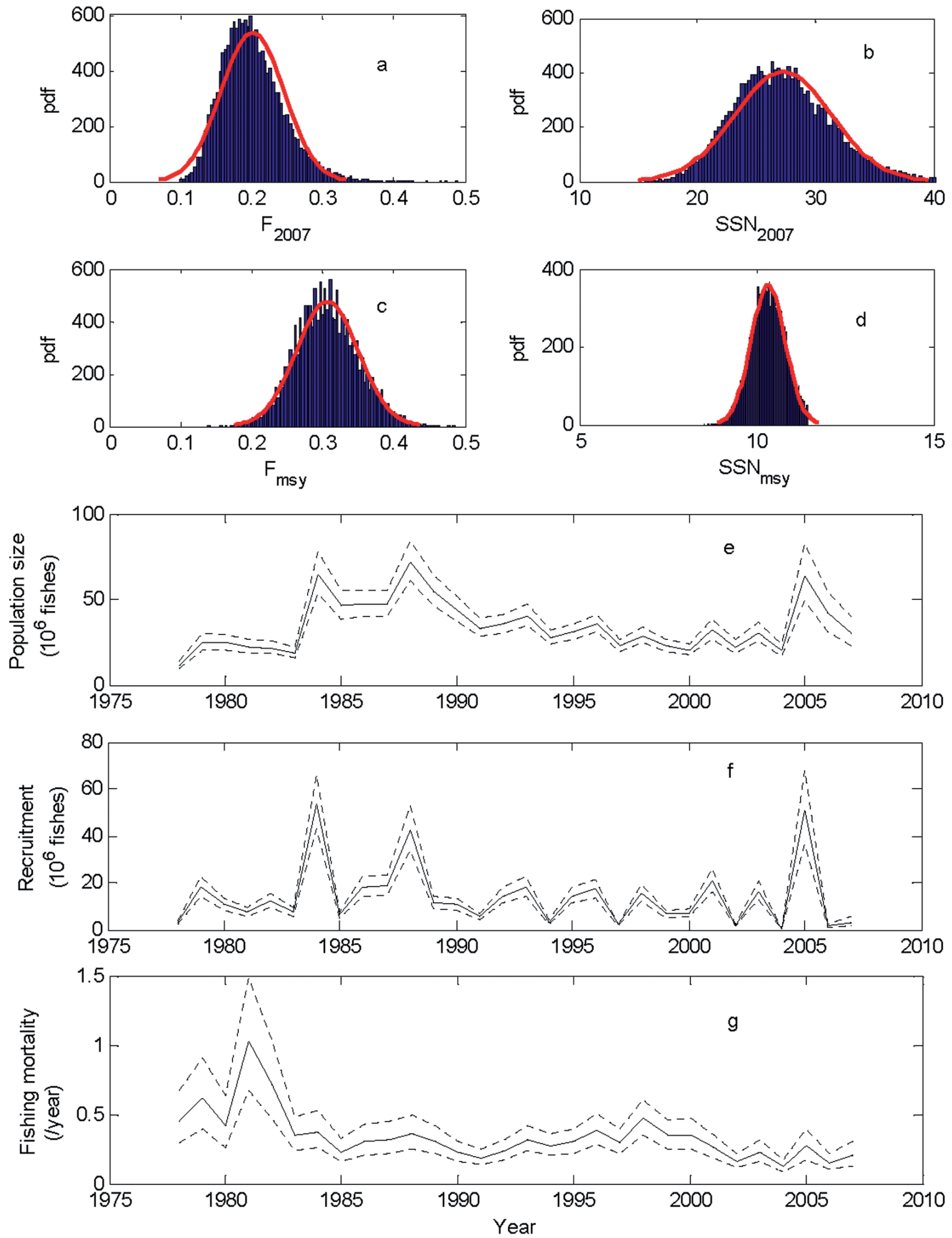

FIG. 4. - Posterior probability distributions of (a) fishing mortality, (b) abundance, (c) estimated $F_{m s v}$, and (d) estimated $N_{m s y}$ for the walleye fishery in 2007. Normal distribution fits (red lines) are overlaid on the plots. Time series of mean and $95 \%$ credible intervals from the posterior distributions of (e) population size, (f) recruitment and (g) fishing mortality. 


\section{RESULTS}

The most probable state of the Lake Erie walleye fishery in 2003 was H1 (51.1\%) for the TLCR (Fig. 2a). There was a 39.3\% (H2) plus $9.6 \%(\mathrm{H} 3)$ chance that the actual state of the fishery lay under the TLCR. Thus, there was no evidence that walleye were overfished, which would have been in areas H4 to H7. In 2007, the most probable state was also in $\mathrm{H} 1(71.0 \%)$ for the TLCR (Fig. 2b). There was a $23.2 \%(\mathrm{H} 2)$ plus $5.8 \%(\mathrm{H} 3)$ chance that the actual state of the fishery lay under the TLCR. Thus, there was no evidence that walleye were overfished, which would have been in areas $\mathrm{H} 4$ to $\mathrm{H} 7$.

The mean estimate of fishing mortality in 2007 was $0.20(\mathrm{CV}=0.22$, based on the normal distribution fit, Fig. 4a). The probability that $F$ exceeded mean $F_{m s y}$ (mean $=0.31, \mathrm{CV}=0.14$; Fig. $4 \mathrm{c}$ ) was $5.7 \%$, and the probability that $F$ exceeded $F_{m s y}$ was $5.8 \%$ (Fig. 2b). The mean estimate of walleye spawning stock population size in 2007 was 27.28 million fish $(\mathrm{CV}=0.15$, Fig. $4 \mathrm{~b})$, with a $0 \%$ chance that the spawning stock population size was lower than $S S$ $N_{m s v}$ (mean=10.35, CV=0.05; Fig. $4 \mathrm{~d}$ and Fig. 2b).

When the analysis shown in Figure 2 for 2007 is extended back over all years in the study, it is apparent that overfishing was happening in many of them (predominant probability in $\mathrm{H} 3$ or $\mathrm{H} 7$ ) albeit without significant repercussions for stock size (mostly low

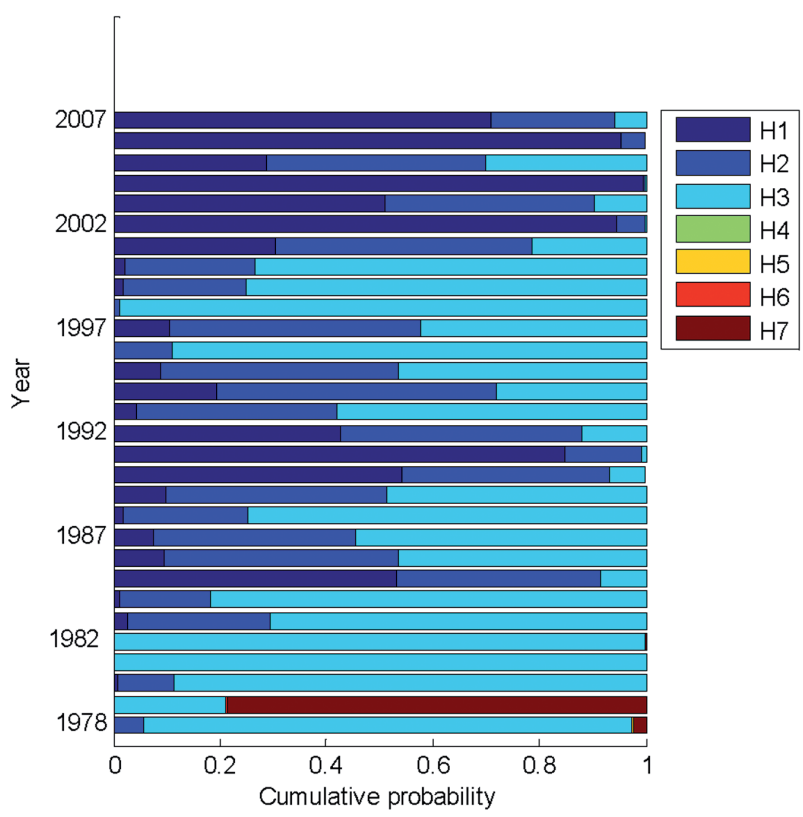

FIG. 5. - Fishery status shown as the probability of the fishery being located in each region in the TLCR for each study year, 1978 to 2007. The 2007 bar corresponds to Figure $2 b$.
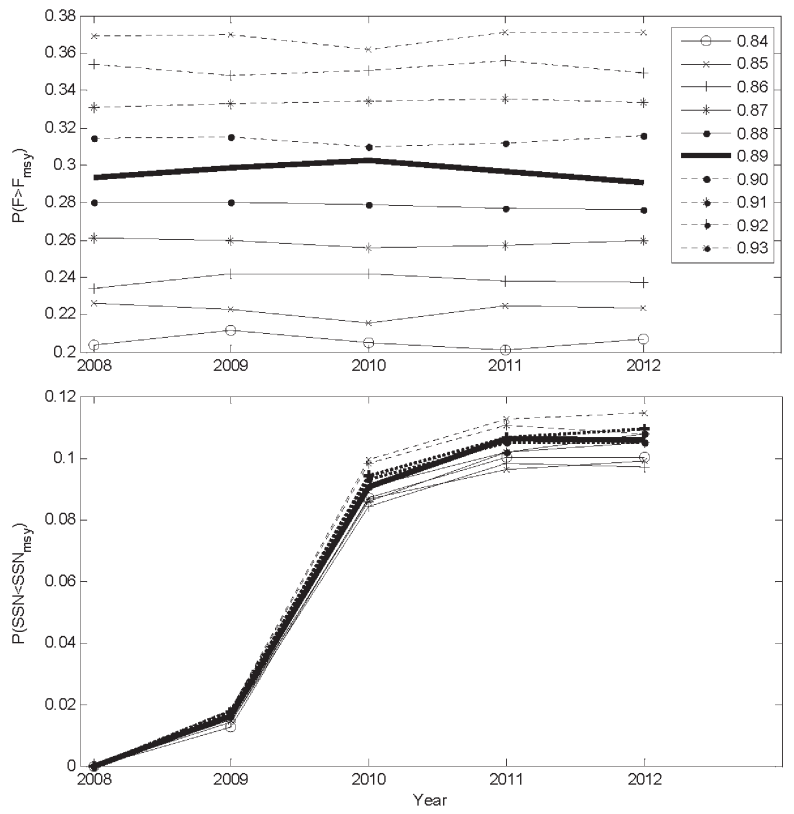

FIG. 6. $-F$ as a fraction of $F_{m s y}$ and their corresponding risks of $P(F>$ $\left.F_{m s y}\right)$ and $P\left(S S N<S S N_{m s y}\right)$. Legend indicates the fraction value used in the projection.

probabilities in $\mathrm{H} 4$ to $\mathrm{H} 7$; Fig. 5). The state of this walleye fishery appears to be heavily influenced by large fluctuations in recruitment (Fig. 4f), suggesting that better recruitment prediction would contribute to a reduction in uncertainty.

A probability distribution for $F$ in the projection was generated in this example fishery and the trajectories of the Bayesian posterior runs were used in the projection, with the management objective $P(F>$ $\left.F_{m s y}\right)=30 \%$ and $P\left(S S N<S S N_{m s y}\right)=80 \%$. The fraction of $F_{m s y}$ of $89 \%$ was the most appropriate value of $F$, which satisfied the management objective (Fig. 6). Time varying fraction values were estimated and were between 89.0 and $89.3 \%$ among the five simulated years if the management objective was fixed to be exactly $P\left(F>F_{m s y}\right)=30 \%$. The estimated $P(F$ $>F_{m s v}$ ) were very close to $30 \%$ with a fixed fraction of $89 \%$ among the 5 projected years, and $P(S S N<$ $S S N_{m s y}$ ) were less than $80 \%$ in all the five projected years. The fishing mortality and the corresponding catch were shown in Figure 7. Projected population size and recruitment further indicated that large variation of recruitment heavily influenced the population projection (Figs. 6 and 7).

When $S S N$ and $F$ were assumed to be estimated from one model, while $S S N_{m s y}$ and $F_{m s y}$ were assumed to be estimated from another model, and the correlation between $F$ and $F_{m s y}$, and the correlation between $S S N$ and $S S N_{m s y}$ were not estimable, we de- 

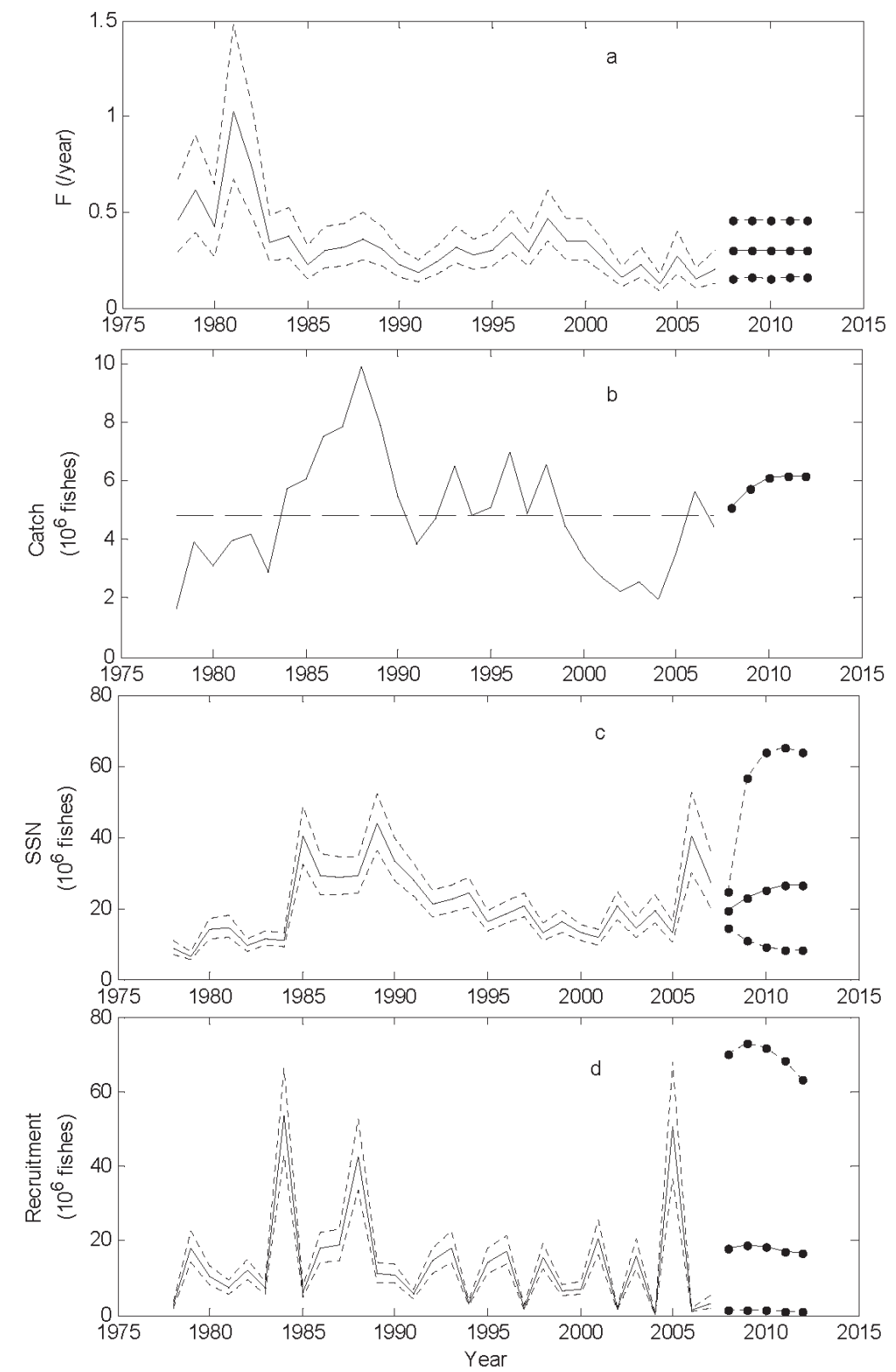

FIG. 7. - Projections of the fishing mortality, population size and catch based on the method shown in Figure 3 (also see the methods section). a): solid line: estimated fishing mortality based on data from 1978 to 2007; dotted lines: posterior $95 \%$ credible interval of fishing mortality; solid line with marker: projected mean fishing mortality for management; dotted lines with marker: $95 \%$ confidence interval of the projected fishing mortality. b): solid line: observed catch from 1978 to 2007; dashed line: mean catch among 1978 to 2007; solid line with marker: projected total allowable catch that matches the example management objective. c): solid line: estimated spawning stock size (SSN) based on data from 1978 to 2007; dotted lines: posterior 95\% credible interval of SSN; solid line with marker: projected mean SSN; dotted lines with marker: 95\% confidence interval of the projected SSN. d): solid line: estimated recruitment based on data from 1978 to 2007; dotted lines: posterior 95\% credible interval of recruitment; solid line with marker: projected mean recruitment; dotted lines with marker: $95 \%$ confidence interval of the projected recruitment.

signed the control rule by assuming that $S S N, S S N_{m s y}$ and $F, F_{m s y}$ are normally distributed. This assumption is based on the estimated posterior distributions from the Bayesian approach (Fig. 4). In this example fishery, if we assume that stakeholders accept risk levels of $P\left(F>F_{m s y}\right)=30 \%$ and $P\left(S S N<S S N_{m s y}\right)=$ $80 \%$, then, according to the current year's $F, F_{m s y}$ and their uncertainty, we would get $P\left(F>F_{m s y}\right)=$
$30 \%$ when the mean of $\left(F / F_{m s y}\right)=0.85$ according to the posterior runs of $F$ and $F_{m s y}$ and the correlation between them. We would get $P\left(S S N<S S N_{m s y}\right)$ $=80 \%$ when the mean of $\left(S S N / S S N_{m s y}\right)=0.88 \mathrm{ac}-$ cording to the posterior runs of $S S N$ and $S S N_{m s y}$ and their correlation. Therefore, A new control rule can be designed: $\mu\left(F / F_{m s y}\right)=0.85$ as the target control $F_{\text {target }}$, and $\mu\left(S S N / S S N_{m s y}\right)=0.88$ as the limit con- 

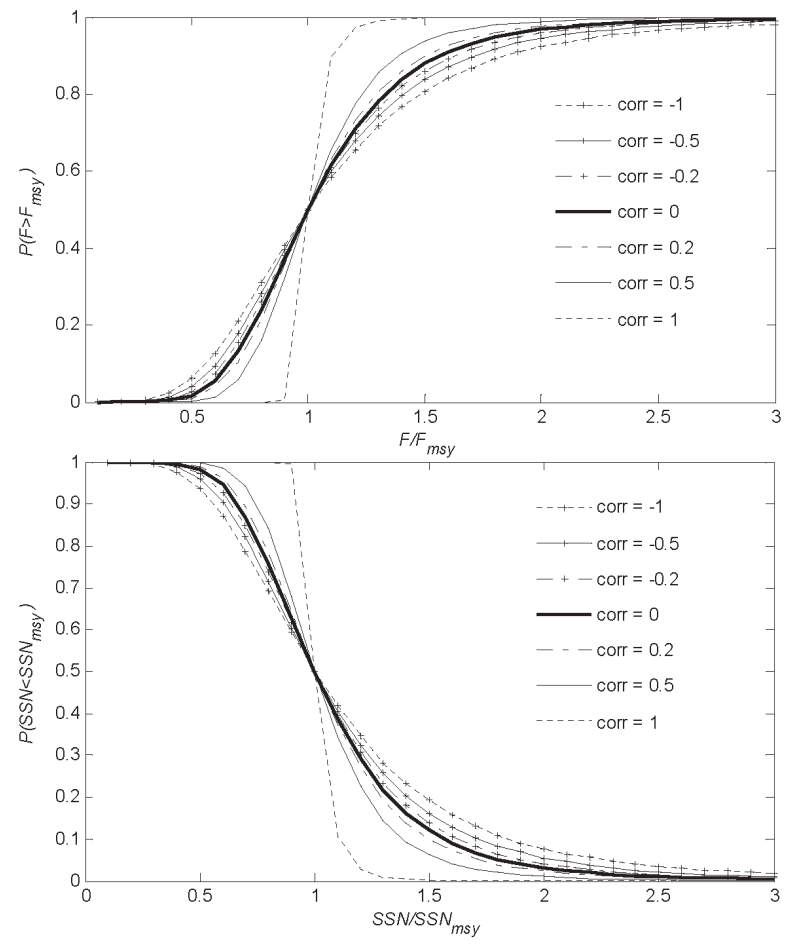

FIG. 8. - Risk of using different target fishing mortalities and $N_{\text {thresh- }}$ old in the designed control rule. To illustrate the influence of correlations between indicators, correlation coefficients between $F$ and $F_{m,}$ and between $S S N$ and $S S N_{m, y}$ vary from -1 to 1 . Uncertainty (CVs here) of $F_{m s y}$ and $S S N_{m s y}$ are from the walleye fishery example.

trol $S S N_{\text {threshold }}$ (Fig. 8). The risk variation when the correlation coefficient varies is shown in Figure 8. Same risk values were estimated according to the projected population size and the fishing mortality that satisfied the management objectives. $\mu\left(F / F_{m s y}\right)$ was between 0.88 and 0.89 among the 5 projected years.

The potential for bias due to relaxation of the assumption of a normal distribution was evaluated. For example, when the $\mathrm{CV}$ of $F$ is set to 0 (and hence $F$ has no 'distribution'), the corresponding risk of $F$ $>F_{m s y}$ is 0.05 when $F=0.62 F_{m s y}$ (Fig. 8a), which is the lower $95 \% \mathrm{CI}$ of the $F$ posterior distribution. When the CV of $S S N$ is set to 0, the corresponding risk of $S S N<S S N_{m s y}$ is 0.95 when $S S N=0.88 S S N_{m s y}$ (Fig. 8b), which is the lower 95\% CI of the $S S N$ posterior distribution. The magnitude of bias can be diagnosed by examining the difference between the empirical values and extreme values such as $\mathrm{CV}=0$.

If the correlation between $F$ and $F_{m s y}$ and that between $S S N$ and $S S N_{m s y}$ are estimable, correlations between biological reference points are easily considered through a bivariate normal distribution (Fig. $8)$. In this study, $F$ and $F_{m s y}$ and $S S N$ (2007) and $S S$ $N_{m s y}$ were weakly correlated (-0.09 and 0.44 respectively in 2007, Fig. 9). To design a control rule for the next year's or next few years' management, we will have to assume no correlations or use the correlations from the study with data up to the current assessment or carry out extra sensitivity studies with a range of correlation possibilities. Bias can be evaluated by comparing the estimated risk when extreme values are used (i.e. using the true distributions of $F$ and $N$ ) with the corresponding estimated risks from the empirical values in Figure 8, and assuming they are normally distributed and uncorrelated (Jiao et al., 2005). For example, in 2007 the estimated risk of $P\left(F>F_{m s y}\right)=5.7 \%$ and $P\left(S S N<S S N_{m s y}\right)=0 \%$ from the Bayesian procedure, which included correlation, but the estimated risk of $P\left(F>F_{m s y}\right)=5.3 \%$ and $P\left(S S N<S S N_{m s y}\right)=0 \%$ from the composite risk assessment approach, which assumed non-correlation. The bias of ignoring correlations between $F$ and $F_{m s y}$ and $S S N$ and $S S N_{m s y}$ was thus very small, i.e. $5.7 \%$ vs. $5.3 \%$ and $0 \%$ vs. $0 \%$. Because the correlation between $S S N$ and $S S N_{m s y}$ was high, its influence on
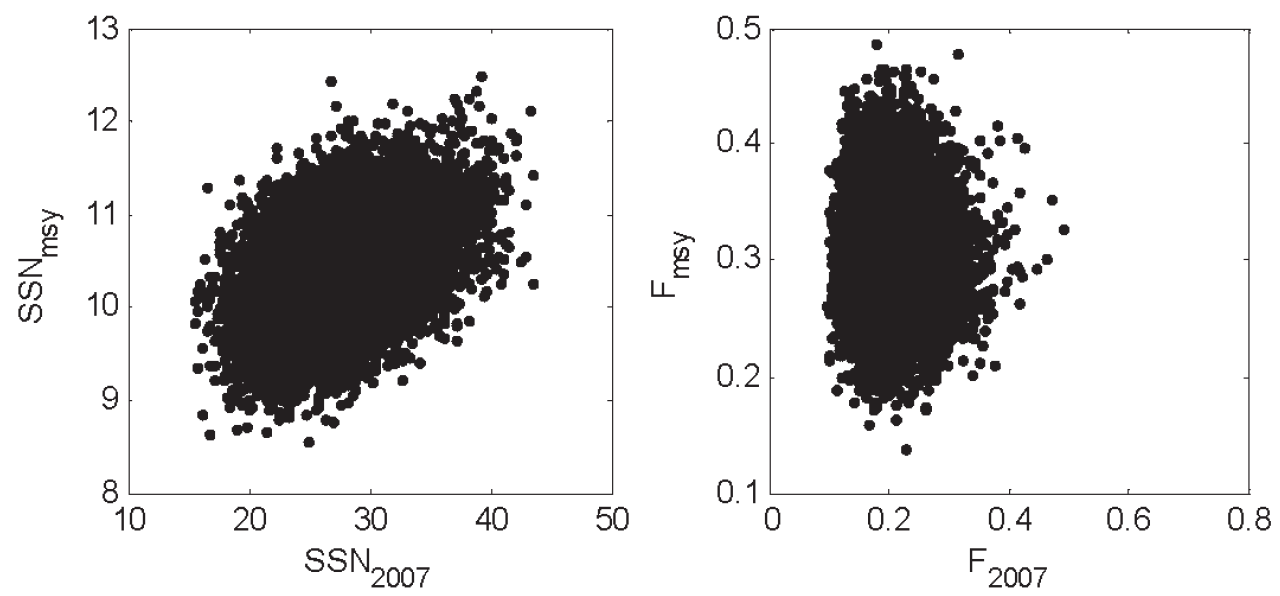

FIG. 9. - Joint posterior distributions of $F$ and $F_{m s y}$, and $S S N$ and $S S N_{m s y}$. 
$P\left(S S N<S S N_{m s y}\right)$ should be higher when the ratio of $S S N / S S N_{m s y}$ is not too small or too large (e.g. between 0.75 and 1.50) than when $S S N / S S N_{m s y}$ is close to 0 or 2 (Fig. 8).

Population projections assuming $F$ and $F_{m s y}$ and $S S N$ and $S S N_{m s y}$ followed normal distributions and with and without correlations were very close to those of population projections using joint Bayesian posterior runs. We did not show the results for brevity.

\section{DISCUSSION}

The approach used here was based on a risk analysis of the control rules, which compares the difference between population abundance and $N_{m s y}$ and between fishing mortality and $F_{m s y}$ or MSY-related $N$ or $F$ based reference points. For example, when population abundance is high, overexploitation will tend to put the fishery status mainly in $\mathrm{H} 3$ for TLCR. On the other hand, when population abundance is low, overexploitation will tend to put the fishery status mainly in $\mathrm{H} 7$ for TLCR. Thus, the implications of the fishery status are very different. When the best estimate of fishery status is in $\mathrm{H} 2$ for TLCR, there may still be a high probability that the population is in $\mathrm{H} 1$ or $\mathrm{H} 3$ because of uncertainty of the fishery status. Further detailed quantification, with more regions sub-dividing the graphical region, can be made to better estimate the risk by including both the uncertainty information on exploitation and population size status.

Our approach provided a convenient way of incorporating the uncertainty of fishing mortality caused by implementation error and estimation error into the design of a control rule based on the real fisheries data and fisheries management. When the joint distributions of the population size, and other parameters such as selectivity and $F_{m s y}$ and $S S N_{m s y}$ are available, such as in this example, the Bayesian posterior runs can be used for projection based on the Bayesian posterior trajectories. When the joint distribution of $F$ and $F_{m s y}$, and $S S N$ and $S S N_{m s y}$ are not available, the probability distributions of $F_{m s y}$ and $S S N_{m s y}$ can be used as the next year's when we design the control rule for management; however, they are independent of the population projection. The posterior distributions of biological reference points $F_{m s y}$ and $S S N_{m s y}$ are robust to the extra years' data. Uncertainty of fishing mortality can be influ-

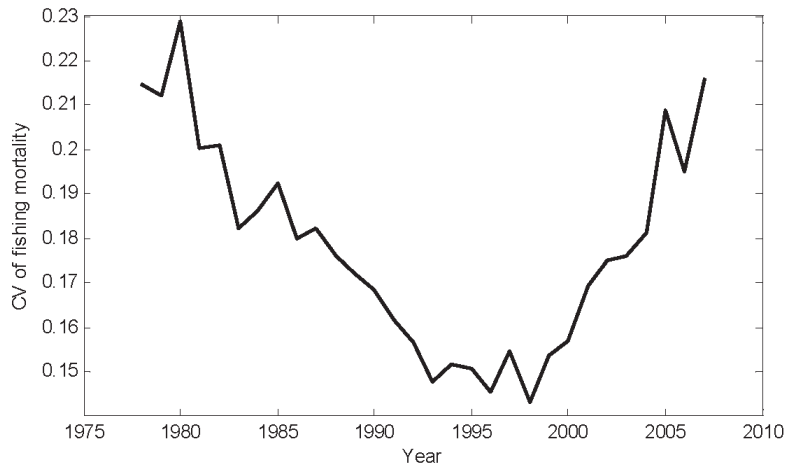

FIG. 10. - CVs of fishing mortality from 1978 to 2007 estimated from the Bayesian statistical catch-at-age model.

enced by many different factors, such as policy on mesh nets, size limit, quota, etc., but it is not totally unpredictable. In this fishery example, we found that uncertainties in the fishing mortality (here, expressed as $\mathrm{CV}$ ) in neighboring years were always close, and the overall CV range was between 18 and 29\% (Fig. 10). For species that are assessed every 3-4 years, uncertainty of fishing mortality ( $\mathrm{CV}$ here) from the average or range of several previous years is suggested when it is used in the population projection.

Determining an acceptable risk level is important when designing the control rule. When considering what an appropriate risk level is for a specific species or stock, the resilience of the fishery to overfishing needs to be considered. In this example, walleye tend to be resilient to ecosystem changes and overexploitation (Munawar et al., 2002). Even though the fishery was overexploited (according to the $F_{m s y}$ reference point) for most of the years (Fig. 5 ), the fishery had highly varied recruitment (Fig. 4) and could rebound to a large population size. For species that are assessed every year, the control rule can be changed every year because of the changes in the uncertainty of $F_{m s y}$ and $F, S S N$ and $S S N_{m s y}$. The approach proposed in this paper calls for a riskspecific adjustment of fishing mortality to a percentage of $F_{m s y}$ such that

$$
F_{\text {threshold }}=\% F_{m s y} \frac{S S N}{S S N_{\text {threshold }}},
$$

and for the adjustment of $S S N_{\text {threshold }}$.

Although this study is based on a statistical catchat-age model, a similar approach can be derived from surplus production models, which are used by many fisheries managers with or without age- or size-structured data. Evaluating bias is important when correlations between $F$ and $F_{m s y}$, and SSN and 
$S S N_{m s y}$ are examined. We recommend including bias in risk assessment and control rule design, as it will be significant when correlations are high.

This study provides a practical approach to explicitly incorporate uncertainty of fishing mortality, of $F$ and $B$-based biological reference points, and of population size in the design and use of control rules in fisheries management. Applying control rules without considering uncertainty has been found to cause distrust among stakeholders (e.g. at the 2007 meeting of the South Atlantic Fishery Management Council). Our proposed method should improve fisheries management in this respect.

Incorporating the uncertainty of the fishery in the control rules can improve understanding and acceptance of the fishery management process among all participants. To quote Koeller (2003): "If you confess your uncertainty verbally you will be ridiculed but if you confess your uncertainty mathematically you will be forgiven". The design of the control rules and the probabilistic presentation of the various status areas associated with the control rules offer a powerful and interpretable way of understanding risk and uncertainty in the fishery. Due to the generality of the approach, we believe that it is a valuable and complementary tool for stock assessment and fisheries management.

\section{ACKNOWLEDGEMENTS}

This research was supported by grants from Virginia Polytechnic Institute and State University, the USDA Cooperative State Research, Education and Extension Service, Hatch Project \#0210510, and the MARFIN NOAA to Y. Jiao, and by the Ontario Commercial Fisheries Association to Y. Jiao. We are grateful to Dr. Andreas Winter and Dr. Mike Errigo for pre-reviewing this manuscript. Special thanks are given to Andy Cook from Lake Erie Management Unit who provided valuable comments on an earlier version.

\section{REFERENCES}

ASMFC (Atlantic States Marine fisheries Commission). - 2002. Amendment 4 to the Interstate Fishery Management Plan for Atlantic Weakfish. Fish. Manag. Rep. No. 39: 1-101pp.

Brodziak, J. and C.M Legault. - 2005. Model averaging to estimate rebuilding targets for overfished stocks. Can. J. Fish. Aquat. Sci., 62(3): 544-562.

Butterworth, D.S. and P.B. Best. - 1994. The origins of the choice of 54\% of carrying capacity as the protection level for baleen whale stocks, and the implications thereof for management procedures. Rep. Int. Whal. Commn., 44: 491-497.

Caddy, J.F. and R. Mahon. - 1995. Reference points for fisheries management. FAO Fish. Tech. Pap., 347.

Chen, Y. and C. Wilson. - 2002. A simulation study to evaluate impact of uncertainty on the assessment of American lobster fishery in the Gulf of Maine. Can. J. Fish. Aquat. Sci., 59(8): 1394-1403.

Chen, Y., Y. Jiao and L. Chen. - 2003. Developing robust frequentist and Bayesian fish stock assessment methods. Fish Fish., 4(2): 105-120.

de Valpine, P. and A. Hasting. - 2002. Fitting population models incorporating process noise and observation error. Ecol. Monogr., 72(1): 57-76.

FAO. - 1995. Precautionary Approach to Fisheries. Part 1: Guidelines on the precautionary approach to capture fisheries and species introductions, United Nations. FAO Fish. Tech. Pap,. $350 / 1$.

Francis, R.I.C.C. and R. Shotton. - 1997. "Risk" in fisheries management: a review. Can. J. Fish. Aquat. Sci., 54(8): 1699-1715.

Garcia S.M. - 1996. The precautionary approach to fisheries and its implications for fishery research, technology and management: An updated review. FAO Fish. Tech. Paper, 350/2.

Gilks, W.R. - 1996. Full conditional distributions. In: W.R. Gilks, S. Richardson and D.J. Spiegelhalter (eds.), Markov Chain Monte Carlo in Practice, pp. 75-78. Chapman and Hall, London, U.K.

Helser, T.E., T. Sharov and D.M Kahn. - 2001. A stochastic decision-based approach to assessing the Delaware Bay blue crab (Callinectes sapidus) stock. In: J.M. Berkson, L.L. Kline and D.J. Orth (eds.), Incorporating uncertainty into fishery models, pp. 63-82. American Fisheries Society Publication, 27. Bethesda, Md.

Hilborn R. - 2002. The dark side of reference points. Bull. Mar. Sci., 70(2): 403-408.

Hilborn, R. - 2006. Fisheries success and failure: the case of the Bristol Bay salmon fishery. Bull. Mar. Sci., 78(3): 487-498.

Hilborn, R. and C. Walters. - 1992. Quantitative Fisheries Stock Assessment: Choice, Dynamics, and Uncertainty. Chapman and Hall, New York.

Hoeting, J.A., D. Madigan, A.E. Raftery and C.T. Volinsky. - 1999. Bayesian model averaging: a tutorial. Stat. Sci., 14(4): 382-417.

Jiao, Y., Y. Chen and J. Wroblewski. - 2005. An application of the composite risk assessment method in assessing fisheries stock status. Fish. Res., 72(2-3): 173-183.

Katsukawa, T. - 2004. Numerical investigation of the optimal control rule for decision-making in fisheries management. Fish. Sci., 70(1): 123-131.

Koeller, P. - 2003. The lighter side of reference points. Fish. Res., 62(1): $1-6$

Lake Erie Walleye Task Group (LEWTG). - 2004. Report of the walleye task group to the standing technical committee. Lake Erie Committee of the Great Lakes Fishery Commission.

Lake Erie Walleye Task Group (LEWTG). - 2008. Report of the walleye task group to the standing technical committee. Lake Erie Committee of the Great Lakes Fishery Commission.

May, R.M., J.R. Beddington, C.W. Clark, S.J. Holt and R.M. Laws. - 1979. Management of multispecies fisheries. Science, 205(4403): 267-277.

McAllister, M.K. and J.N. Ianelli. - 1997. Bayesian stock assessment using catch-age data and the sampling-importance-resampling algorithm. Can. J. Fish. Aquat. Sci., 54(2): 284-300.

Millar, R.B. and R. Meyer. - 2000. Non-linear state-space modeling of fisheries biomass dynamics by using Metropolis-Hastings within-Gibbs sampling. Appl. Stat., 49(3): 327-342.

Munawar, M., I.F. Munawar, R. Dermott, H. Niblock and S. Carou. - 2002. Is Lake Erie a resilient ecosystem? Aquat. Ecosyst. Health Manage., 5(1): 79-93.

Patterson, K., R. Cook, C. Darby, S. Gavaris, L. Kell, P. Lewy, B. Mesnil, A. Punt, V. Restrepo, D.W. Skagen and G. Stefansson. - 2001. Estimating uncertainty in fish stock assessment and forecasting. Fish Fish., 2(2): 125-157.

Punt, A.E. and R. Hilborn. - 1997. Fisheries stock assessment and decision analysis: the Bayesian approach. Rev. Fish. Biol. Fish., 7(1): 35-63.

Quinn, T. and R.B. Deriso. - 1999. Quantitative fish dynamics. Oxford University Press, Oxford, UK. 
Restrepo, V.R., and J.E. Powers. - 1999. Precautionary control rules in US fisheries management: specification and performance. ICES J. Mar. Sci., 56(6): 846-852.

Restrepo, V.R., G.G. Thompson, P.M. Mace, W.L. Gabriel, L.L. Wow, A.D. MacCall, R.D. Methot, J.E Powers, B.L. Taylor, P.R. Wade and J.F. Witzig. - 1998. Technical guidance on the use of precautionary approaches to implementing National Standard 1 of the Magnuson-Stevens Fishery Conservation and Management Act. NOAA Tech. Memora. NMFS-F/SPO- 31.

Roughgarden, J. and F. Smith. -1996. Why fisheries collapse and what to do about it? Proc. Natl. Acad. Sci. USA., 93(10): 5078-5083.

Rubin, D.B. - 1988. Using the SIR algorithm to simulate posterior distributions. In: J. Bernardo, M. DeGroot, D.V. Lindley and A.F.M. Smith (eds.), Bayesian Statistics, 3, pp. 395-402. Oxford University Press, Oxford.

SAFMC (South Atlantic Fisheries Management Council). - 2005. SEDAR stock assessment for Black Sea Bass. Beaufort, North Carolina.
Shepherd, J.G. - 1982. A versatile new stock-recruitment relationship for fisheries, and the construction of sustainable yield curves. I. Cons. Int. Explor. Mer., 40(1): 67-75.

Smith, A.F.M. and A.E. Gelfand. - 1992. Bayesian statistics without tears: A sampling-resampling perspective. Amer. Stat., 46(2): 84-88.

Spiegelhalter, D.J., A. Thomas, N. Best and D. Lunn. - 2004. WinBUGS user manual (version 1.4.1). MRC Biostatistics Unit, Cambriage, U.K.

Sunstein, C.R. - 2005. Cost-benefit analysis and the environment? Ethics, 115(2): 351-385.

Thompson, G.G. - 1993. A proposal for a threshold stock size and maximum fishing mortality rate. Can. Spec. Publ. Fish. Aquat. Sci., 120: 303-320.

Scient. ed.: F. Maynou.

Received March 12, 2009. Accepted October 13, 2009.

Published online March 19, 2010. 